
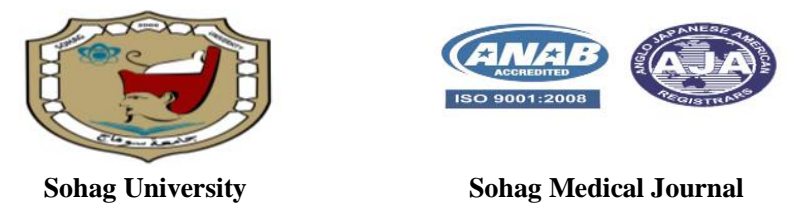

Sohag Medical Journal

Faculty of Medicine

\title{
Role of metabolic Positron Emission Tomography parameters in liver metastasis from Colon and rectal cancer.
}

\author{
Doaa Ibrahim*, Mohammed Soliman**, Esraa Elkholy***, Wafaa \\ Abdelhameid*, \\ * Nuclear medicine unit, faculty of medicine, Sohag university \\ ** Oncology department, faculty of medicine, Sohag university \\ ***Nuclear medicine unit, faculty of medicine, Cairo university
}

\begin{abstract}
Introduction: Colon and rectal cancers are common malignancies all over the world. Although curative resection of the primary lesion improves survival, still less than half the number of patients, die from colon and rectal cancer mostly those with distant metastases.

The most common site of distant metastasis is considered the liver. This is true regarding many types of malignancies and of especial consideration in the colon and rectal malignancies.

PET/CT is a very valuable tool in the follow-up of colorectal cancer patients, SUVmax is the most commonly used PET parameter and represents the voxel of the highest activity within the ROI. However, other parameters as MTV and TLG gain an interest in recent studies.

Aim of the work: To highlight the value of quantitative PET/CT parameters in colorectal patients with liver metastasis.
\end{abstract}

Keywords : Colon rectal liver metastasis, PET/CT, SUVmax, MTV, TLG

\section{Introduction:}

Colon and rectal cancer are very common malignancies all over the world. However, less than half of the patients still die from their disease especially those with distant metastases, even after curative surgery ${ }^{(1)}$.

The liver is considered the most affected site with blood spreading for many neoplasms. Hepatic metastases occur in a wide variety of neoplasms, especially with colon and rectal cancer ${ }^{(2)}$. hepatic metastases from the colon or rectal cancers were considered terminal in the past, but recently, it is believed that it can be cured with surgery, which improves the rate of survival for these patients by up to $30 \%-50 \%$. Als$\mathrm{o}$, when liver metastases from colorectal cancer are not resectable, giving chemotherapy before surgical intervention can allow its resection and this strategy can improve the survival rate of these patients ${ }^{(3)}$.

\section{Epidemiology of colorectal cancer}

Colon and rectal cancers are common in developed countries. with males are more affected than females ${ }^{(4)}$. Although the incidence of colon and rectal cancers decreased more cases are reported in younger age below 50 years old $^{(5)}$.

In Egypt colon cancer is ranked as seventh cancer, although, ninety percent of colon cancer occurs after the age of fifty-five, in Egypt, there is a high incidence of colon cancer at a young age with an incidence rate of thirty-eight percent in individuals under the age of 
forty $^{(6 a n d 7)} .70$ and 72 are the median ages in males and females respectively ${ }^{(8)}$, proximal colon is more likely to be affected in females and old aged patients, while in males, the distal colon is more affected ${ }^{(9)}$.

\section{Types of colon and rectal cancer according to histological examina- tion}

Typical adenocarcinoma represents $\mathrm{m}$ ost cases of colon and rectal cancer, other types of adenocarcinomas as mucinous and signet ring types are rare. The prognosis of typical adenocarcinoma is considered the best while signet ring cell type has a poor prognosis, the prognosis of mucinous adenocarcinoma is unclear. Some studies reported poor outcomes in patients with mucinous adenocarcinoma while other studies reported the opposite results ${ }^{(4-10)}$. Although most CRC develops from adenomatous polyps, nearly a tenth of $\mathrm{p}$ olypoid adenomas proceed to invasive cancer $^{(4,11)}$.

Factors like gene mutations, epigenetic alterations, and local inflammatory changes have a respectable role in the transformation of benign polyp to malignant disease ${ }^{(12)}$.

The rectum and sigmoid colon may be affected with gastrointestinal stromal tumors although the stomach is the $\mathrm{m}$ ost common site for GIST in the gastrointestinal system. Mostly they arise transmural with intraluminal or outward bulging. Rarely, they can present as subserosal lesions. The site, size, and mitotic activity of GIST are included in the risk assessment of patients (13).

Diffuse large B-cell lymphoma (DLB$\mathrm{CL}$ ) is the most common lymphoma subtype affecting the colon with the cecum and rectosigmoid are the two most common sites of involvement. Follicular lymphoma also can be encountered in the colon mostly occurs in the ileocecal and ascending colon. It can present as multiple mucosal polyps up to $1 \mathrm{~cm}$. Burkitt's lymphoma also had reported in the rectum and ileocecum, although rare, it is an aggressive type of B-cell lymphomas. Leukemias also had been reported in the right colon $^{(14)}$.

\section{Role of Radiological imaging}

1- Diagnosis: radiological diagnosis is very important as most patients are asymptomatic especially in the early stages. While less than a third may present with symptoms of complications as bowel perforation, obstruction, or gastrointestinal bleeding mainly in elderly patients and when occurring they indicate poor prongosis. ${ }^{(15)}$.

2- Local Staging: assessment of lymph node status before surgery is critical; in addition to having a prognostic value, it also helps in deciding treatment options for colorectal cancer patients. CT is commonly used for lymph nodes evaluation because it is a quick examination for both chest and abdomen ${ }^{(16)}$.

The size of the lymph node is the used criteria to determine pathologic lymph nodes. Any lymph node more than $1 \mathrm{~cm}$ in its short-axis diameter is reported as abnormal. But the upper limit of benign LNs differs according on their location and the type of tumor. Also, malignancy may occur in lymph nodes less than $1 \mathrm{~cm}$. So, $\mathrm{N}$ staging according to the size of the lymph nodes sometimes is misleading ${ }^{(12)}$.

3- Assessment of response: PET/CT images are interpreted qualitatively and quantitatively analyzed. ${ }^{(17)}$ The fundamental principle of assessing tumor response is to assess both size and metabolic activity of all lesions in baseline scan before starting a new therapy so the response to therapy can be quantified by calculating the relative change from the 
baseline. $^{(18)}$. Also, FDG PET/CT provides both quantitative and qualitative data on the metabolism of tumor cells, which is very sensitive for the effect of treatment, and so it is considered a very valuable tool $\mathrm{f}$ or treatment response evaluation ${ }^{(19)}$.

\section{Liver metastasis}

Hepatic metastases affect approximately $50 \%$ of patients with colon and rectal cancers ${ }^{(20)}$. hepatic resection became popular in the last twenty years due to the improvement of surgical tools with current mortality risks well below 5\% in high-volume centers. But the percent of colorectal liver metastatic patients who are a candidate for liver resection is not exceeding $20 \%(21)$. This is either due to the status of their hepatic or extrahepatic disease. To further improve survival, criteria for selecting patients is very important with general patient factors as his fit-ness to this major surgery, presence of comorbidities, and hepatic factors which can be summarized by the ability to resect with negative margin (R0), whilst maintaining an adequate future liver remnant (FLR) with good vascular supply and biliary drainage (22).

Palliative chemotherapy is considered for patients who are not a candidate for hepatic resection and improves the rate of their survival by about $10 \%$ (23).

Liver transplantation provides the longest overall survival reported in colon and rectal cancer patients with nonresectable hepatic metastases ${ }^{(24)}$.

\section{Quantitative PET parameters}

${ }^{18} \mathrm{~F}$-FDG PET/CT is a sensitive technique in the detection of liver metastases from colon cancer, its sensitivity was reported to be $84 \%$ for lesions more than $10 \mathrm{~mm}$ while only $16 \%$ for lesions less than $10 \mathrm{~mm}$. However, in compareson to MRI and CT, PET had lower sensitivity, but a higher specificity ${ }^{(30)}$.

1- Standardized uptake value SUVmax
The standardized uptake value (SU$\mathrm{V})$ is the most commonly used semi-quantitative parameter and is calculated by the ratio of activity/unit volume at the region of interest to the activity/unit for whole-body volume $^{(25)}$. there are many different formulas for SUV and SUVmax is the most commonly used one and represents the voxel of the highest activity within the ROI. So, SUVmax does not represent the whole tumor metabolic burden because the value is from only one voxel ${ }^{(26)}$.

There is increased interest in using quantitative parameters in prognosis assessment of many solid tumors especially those obtained from initial PET/CT scan. Although the SUVmax has been the most widely studied parameter, other parameters as metabolic tumor volume and total lesio$\mathrm{n}$ glycolysis gain an interest in recent studies ${ }^{(24)}$.

2- Metabolic tumor volume is a measurement of the tumor volume with high metabolic activity (16). MTV has been suggested to be a predictive marker for survival in the colon and rectal cancer patients ${ }^{(27)}$

3- Total lesion glycolysis represents the product of the mean SUV and the $\mathrm{MTV}^{(28)}$. it is preferred over both the number and maximum intensity of lesions because it considers both parameters while taking into account the level of glucose accumulation within the total volume of all the regions of interest ${ }^{(29)}$.

Lesions with low MTV and TLG are considered to have a better prognosis and are better prognostic indicators than conventional semiquantitative metrics (SUVmax, SUVmean, SUVpeak, and $T / B-r a t i o)$ to predict survival in these patients ${ }^{(21)}$.

\section{List of abbreviations:}

CRC: colorectal cancer

CRLM: colorectal liver metastasis 
DLBCL:diffuse large B-cell lymphoma

FDG: fluorodeoxyglucose

FLR: future liver remnant

GIST: gastrointestinal stromal tumors

MTV: metabolic tumor volume

PET/CT: Positron Emission Tomography hybrid with computed tomography

SUV: standard uptake value

TLG: total lesion glycolysis

\section{References:}

1. $\mathrm{Xu}, \mathrm{J}$., et al. "The significant value of predicting prognosis in patients with colorectal cancer using 18F-FDG PET metabolic parameters of primary tumors and hematological parameters." Annals of Nuclear Medicine. 2019; 33(1) (Shim, 2018 \#55): 32-38.

2. Tam, H. H., et al. "The role of routine clinical pretreatment 18F-FDG PET/CT in predicting the outcome of colorectal liver metastasis." Clin Nucl Med. 2015; 40(5): e259-264.

3. Shim JR, Lee SD, Han SS, Lee SJ, Lee DE, Kim SK, Kim SH, Park SJ, Oh JH. Prognostic significance of 18F-FDG $\mathrm{PET} / \mathrm{CT}$ in patients with colorectal cancer liver metastases after hepatectomy. Eur J Surg Oncol. 2018 May;44(5):670-676. DOI: 10.1016/j.ejso.2018.01.243. Epub 2018 Feb 6. PMID: 29459018.

4. Rawla, P., et al. "Epidemiology of colorectal cancer: incidence, mortality, survival, and risk factors." Przeglad gastroenterologiczny. 2019; 14(2): 89103.

5. Win, A. K., et al. Early-Age-of-Onset Colorectal Carcinoma: An Emerging Public Health Issue. Management of Hereditary Colorectal Cancer: A Multidisciplinary Approach. J. G. Guillem and G. Friedman. Cham, Springer International Publishing. 2020; 1-9.

6. Brand Bateman L, Khamis S, Abdelmoneim S-E, Arafat W, Fouad MN, Khamis Y, Omar A, Abdelmoneim RS, Scarinci I. Designing an Effective Colorectal Cancer Screening Program in Egypt: A
Qualitative Study of Perceptions of Egyptian Primary Care Physicians and Specialists. The oncologist. 2020;25(10):e1525-e1531.

7. Metwally, I.H., Shetiwy, M., Elalfy, A.F., Abouzid, A., Saleh, S.S., Hamdy, M., 2018. Epidemiology and survival of colon cancer among Egyptians: a retrospective study. Journal of Coloproctology 38, 024-029.. doi:10.1016/j.jcol.2017.09.418

8. Glover, M., et al. "Epidemiology of Colorectal Cancer in Average Risk Adults 20-39 Years of Age: A Population-Based National Study." Digestive Diseases and Sciences. 2019; 64(12): 3602-3609.

9. Murphy, N., et al. "Heterogeneity of Colorectal Cancer Risk Factors by Anatomical Subsite in 10 European Countries: A Multinational Cohort Study." Clin Gastroenterol Hepatol. 2019; 17(7): 1323-1331 e1326

10.Wu, X., et al. "Prognoses of different pathological subtypes of colorectal cancer at different stages: A population-based retrospective cohort study." BMC Gastroenterology. 2019,19(1): 164 .

11.Johnston, L. and F. Carey (2020). "Pathology of colorectal polyps and cancer." Surgery (Oxford) 38(1): 1217.

12.Grady, W. M. and S. D. Markowitz. "The molecular pathogenesis of colorectal cancer and its potential application to colorectal cancer screening." Digestive Diseases and Sciences. 2015; 60(3): 762-772.

13.Pazhou, M. K. and E. A. Montgomery. Pathological Evaluation, Classification, and Staging of Colorectal Cancers. Textbook of Gastrointestinal Oncology. S. Yalcin and P. A. Philip. Cham, Springer International Publishing. 2019; 37-54.

14.Galletto, A., et al. Complicated Colorectal Cancer: Role of Imaging. Emergency Surgical Management of Colorectal Cancer. N. de'Angelis, S. Di 
SOHAG MEDICAL JOURNAL

Vol. 25 No. 3 July 2021
Saverio and F. Brunetti. Cham, Springer International Publishing. 2019; 55-68.

15. Yang, Z., et al. "Preoperative Diagnosis of Regional Lymph Node Metastasis of Colorectal Cancer with Quantitative Parameters from Dual-Energy CT." American Journal of Roentgenology. 2019; 213(1): W17-W25.

16.Kim, S. H., et al. "Predictive Value of [18F] FDG PET/CT for Lymph Node Metastasis in Rectal Cancer." Scientific Reports. 2019; 9(1): 4979.

17. Kochhar, R. and P. Manoharan. Role of FDG PET-CT in Colorectal Cancer. Comprehensive Rectal Cancer Care. M. Kwaan and A. Zbar. Cham, Springer International Publishing. 2019; 57-84.

18.Kuruva, M., et al. "Multivariate analysis of various factors affecting background liver and mediastinal standardized uptake values." Indian journal of nuclear medicine: IJNM: the official journal of the Society of Nuclear Medicine, India. 2012; 27(1): 20-23.

19.Pinker, K., et al. "Evaluating tumor response with FDG PET: updates on PERCIST, comparison with EORTC criteria and clues to future developments." European Journal of Nuclear Medicine and Molecular Imaging. 2017; 44(Suppl 1): 55-66.

20.Bang, J.-I., et al. "Comparison of Quantitative Methods on FDG PET/CT for Treatment Response Evaluation of Metastatic Colorectal Cancer." Nuclear medicine and molecular imaging. 2017; 51(2): 147-153.

21.Great, H., et al. "The prognostic value of $18 \mathrm{~F}-\mathrm{FDG} \mathrm{PET} / \mathrm{CT}$ prior to liver transplantation for nonresectable colorectal liver metastases." European Journal of Nuclear Medicine and Molecular Imaging. 2018; 45(2): 218225.

22.Selzner, M., et al. "Does the novel PET/CT imaging modality impact on the treatment of patients with
Doaa Ibrahim

metastatic colorectal cancer of the liver?" Annals of surgery. 2004; 240(6): 1027-1036.

23.Mattar, R. E., et al. "Preoperative selection of patients with colorectal cancer liver metastasis for hepatic resection." World journal of gastroenterology. 2016; 22(2): 567581.

24.Dueland, S., et al. "Selection criteria related to long-term survival following liver transplantation for colorectal liver metastasis." American Journal of Transplantation. 2020; 20(2): 530-537.

25.Dueland, S., et al. "Survival Following Liver Transplantation for Patients with Nonresectable Liver-only Colorectal Metastases." Annals of Surgery. 2020; 271.( ( )

26.Ulster, G. A. Chapter 2 - FDG PET/CT Performance and Reporting. Fundamentals of Oncologic PET/CT. G. A. Ulster. 2019; Elsevier: 5-8

27.Vanderhoek, M., et al. "Impact of the Definition of Peak Standardized Uptake Value on Quantification of Treatment Response." Journal of Nuclear Medicine. 2012; 53(1): 4-11.

28.McDonald, J. E., et al. "Assessment of Total Lesion Glycolysis by \&lt; sup\&gt;18\&lt;/sup\&gt; F $\quad$ FDG PET/CT Significantly Improves Prognostic Value of GEP and ISS in Myeloma." Clinical Cancer Research. 2017; 23(8): 1981.

29.I'm, H.-J., et al. "Current Methods to Define Metabolic Tumor Volume in Positron Emission Tomography: Which One is Better?" Nuclear Medicine and Molecular Imaging. 2018; 52(1): 5-15

30.Tsili AC, Alexiou G, Naka C, Argyropoulou MI. Imaging of colorectal cancer liver metastases using the contrast-enhanced US, multidetector CT, MRI, and FDG PET/CT: a meta-analysis. Acta Radiologica. 2021;62(3):302-312. doi:10.1177/0284185120925481 